\title{
Combination Assessment of Clinical Complete Response of Patients With Rectal Cancer Following Chemoradiotherapy With Endoscopy and Magnetic Resonance Imaging
}

\author{
Hye Mi Ko ${ }^{1}$, Yo Han $\mathrm{Choi}^{2}$, Jeong Eun Lee ${ }^{3}$, Kyung Ha Lee ${ }^{1}$, Ji Yeon Kim ${ }^{1}$, Jin Soo Kim ${ }^{1}$ \\ ${ }^{1}$ Department of Surgery, Chungnam National University School of Medicine, Daejeon; ${ }^{2}$ Department of Surgery, Chungcheongnam-do Seosan \\ Medical Center, Seosan; ${ }^{3}$ Department of Radiology, Chungnam National University School of Medicine, Daejeon, Korea
}

Purpose: The response to neoadjuvant chemoradiotherapy (CRT) for rectal cancer can be assessed using digital rectal examination, endoscopy and magnetic resonance imaging (MRI). Precise assessment of clinical complete response (CR) after CRT is essential when deciding between optimizing surgery or organ-preserving treatment. The objectives of this study were to correlate the CR finding in endoscopy and MRI with pathologic CR and to determine the appropriate approach for combining endoscopy and MRI to predict the pathologic CR in patients with rectal cancer after neoadjuvant CRT.

Methods: This retrospective cohort study included 102 patients with rectal cancer who underwent endoscopy and MRI at 2-4 weeks after CRT. We assigned a confidence level (1-4) for the endoscopic and MRI assessments. Accuracy, sensitivity, and specificity were analyzed based on the endoscopy, MRI, and combination method findings. Diagnostic modalities were compared using the likelihood ratios.

Results: Of 102 patients, 17 (16.7\%) had a CR. The accuracy, sensitivity, and specificity for the prediction CR of endoscopy with biopsy were $85.3 \%, 52.9 \%$, and $91.8 \%$, while those of MRI were $91.2 \%, 70.6 \%$, and $95.3 \%$, and those of combined endoscopy and MRI were $89.2 \%, 52.9 \%$, and $96.5 \%$, respectively. No significant differences were noted in the sensitivity and specificity of any each modality. The prediction rate for CR of the combination method was $92.6 \%$ after the posttest probability test.

Conclusion: Our study demonstrated that combining the interpretation of endoscopy with biopsy and MRI could provide a good prediction rate for CR in patients with rectal cancer after CRT.

Keywords: Rectal neoplasms; Chemoradiotherapy; Complete response; Endoscopy; Magnetic resonance imaging

\section{INTRODUCTION}

Neoadjuvant chemoradiotherapy (CRT) for advanced rectal cancer is considered to be a standard therapeutic strategy because it

Received: August 24, 2018 - Accepted: October 15, 2018

Correspondence to: Jin Soo Kim, M.D.

Department of Surgery, Chungnam National University School of Medicine, 282 Munhwa-ro, Jung-gu, Daejeon 35015, Korea

Tel: +82-42-280-8383, Fax: +82-42-257-8024, E-mail: jskim7562@gmail.com ORCID code: https://orcid.org/0000-0002-3362-574X

(C) 2019 The Korean Society of Coloproctology

This is an open-access article distributed under the terms of the Creative Commons Attribution NonCommercial License (http://creativecommons.org/licenses/by-nc/4.0) which permits unrestricted noncommercial use, distribution, and reproduction in any medium, provided the original work is properly cited. decreases the incidence of local recurrence, increases sphincter preservation rate, and improves both the possibility of tumor resection and the survival rate [1-3]. Moreover, approximately $15 \%-25 \%$ of patients who received curative resection after CRT had no viable tumor cells in the resected specimen, otherwise known as complete remission (CR) [4].

Patients with CR following neoadjuvant CRT have been shown to have better oncologic outcomes $[5,6]$. Some investigators have even proposed the "watch-and-wait" strategy for patients with clinical CR after CRT [7]. Given the increasing interest in organpreserving treatment through local excision or a non-operative strategy (watch-and-wait), researchers look forward to finding a reliable treatment strategy in patients with clinical CR [8]. Precise assessment of clinical CR after CRT for patients with rectal cancer 
is essential for establishing such a reliable treatment strategy.

Habr-Gama et al. [7] first described a non-operative "watchand-wait” strategy for patients with clinical CR after CRT in 2004. This study group emphasized the use of digital rectal examination (DRE) and endoscopic findings to identify clinical CR [9]. Additionally, other previous studies have emphasized the importance of endoscopic evaluation of clinical CR $[8,10]$. However, the definition of clinical CR is indeterminate and varies among investigators. In addition, the endoscopic evaluation provides information on only the mucosal status and not the deeper layers such as muscle and mesorectum. On the contrary, magnetic resonance imaging (MRI) can provide more information on the assessment of CR through the whole layer of the rectum. The limitation of MRI is that postirradiation for rectal cancer causes tumor shrinkage and fibrosis. As a result, it is difficult to distinguish the residual tumor from fibrosis using the standard T2-weighted MRI (T2WMRI) when tumor response after CRT is excellent [11]. We postulated that the diagnostic performance might improve if a combined endoscopy and MRI interpretation was performed. Therefore, the objectives of this study were to correlate the CR finding on endoscopy and MRI with pathologic CR and determine the appropriate approach for combining endoscopy and MRI to predict pathologic CR in patients with locally advanced rectal cancer after neoadjuvant CRT.

\section{METHODS}

\section{Patients}

The medical records, endoscopic findings, and MRI images of patients who underwent surgery for rectal cancer after neoadjuvant CRT between November 2010 and October 2016 were reviewed retrospectively. Inclusion criteria were as follows: (1) histologically confirmed adenocarcinoma within $12 \mathrm{~cm}$ from the anal verge, (2) locally advanced disease (clinical T3, T4, or node-positive) without distant metastasis based on radiologic examination, and (3) neoadjuvant CRT. None of the patients had previous pelvic irradiation or chemotherapy. This retrospective study was approved by Institutional Review Board of Chungnam National University Hospital (approval number, 2017-02-065) and the need for informed consent was waived.

The initial evaluation consisted of complete history taking, physical examination, complete blood count, serum biochemistry and carcinoembryonic antigen (CEA) tests, colonoscopy, chest computed tomography (CT), abdominopelvic CT, and MRI. Additionally, positron emission tomography was performed in select instances of uncertain distant metastasis by CT. Rectal tumor was classified as lower $(\leq 4 \mathrm{~cm}$ from the anal verge), middle $(4-8 \mathrm{~cm})$ or upper $(8-12 \mathrm{~cm})$ according to location. Restaging was performed at 2-4 weeks after completion of CRT and consisted of DRE, flexible sigmoidoscopy, complete blood count, serum biochemistry and CEA tests, abdominopelvic CT, and MRI.

Radiation therapy was delivered to the whole pelvis using a 3- or 4-field approach at a total dose of 50.4 Gy. Radiation was administered 5 times per week with a daily fraction of $1.8 \mathrm{~Gy}$ for over 5 weeks, and a boost of $5.4 \mathrm{~Gy}$ was added to the tumor bed. Oral capecitabine at a dose of $825 \mathrm{mg} / \mathrm{m}^{2}$ was administered concomitantly with radiotherapy twice daily for 6 weeks. Postirradiated specimens were evaluated for tumor differentiation, depth of tumor invasion, lymph node metastasis, and tumor regression grade (TRG). The evaluation of TRG evaluation was classified into 4 grades: grade 1 , complete regression; grade 2 , single cells or small groups of cancer cells (moderate response); grade 3 , residual cancer outgrown by fibrosis (minimal response); and grade 4, minimal or no tumor response and extensive residual cancer (poor response). Pathologic CR was identified as the absence of viable tumor cells in the entire specimen.

\section{Assessment of endoscopy}

Enrolled patients underwent flexible sigmoidoscopy (Olympus Medical Systems, Tokyo, Japan) of the rectum after a sodium phosphate enema. White light imaging was used to interpret the response to CRT. Endoscopic evaluation after CRT was performed by a surgeon (JSK). CR was defined as scarring, telangiectasia, and erythema on endoscopy. Potential CR was defined as a shallow ulcer with a regular border (Fig. 1). A biopsy was performed in cases of CR or potential CR. Biopsy was performed in 19 cases, and only 3 cases were revealed to be high grade dyspla-
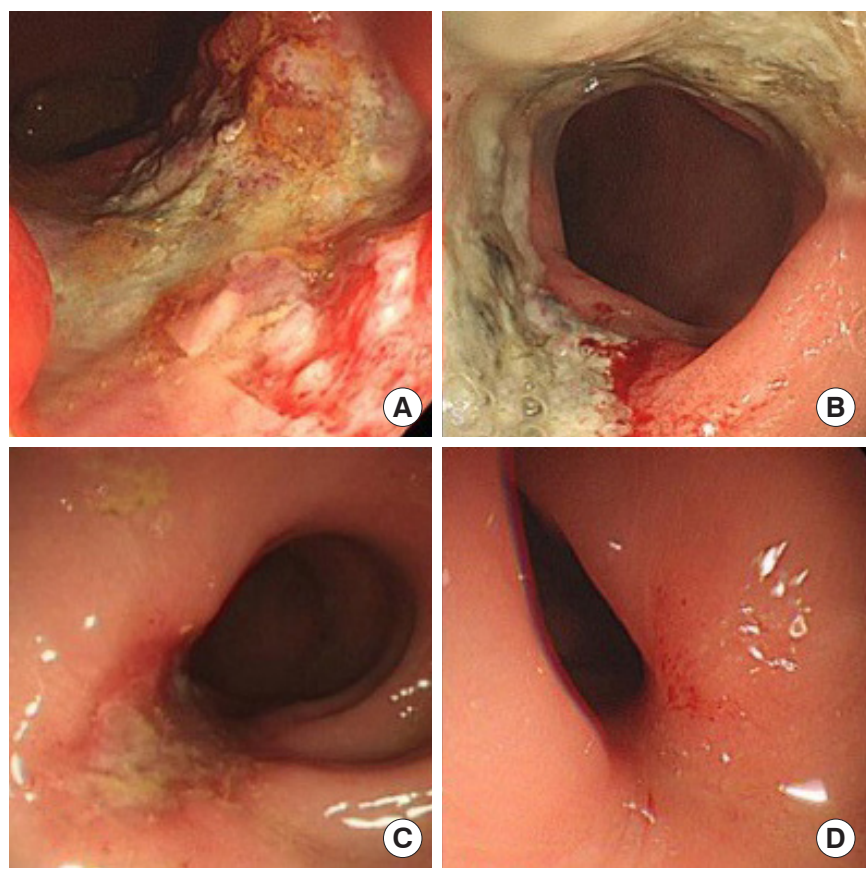

Fig. 1. Response assessment with endoscopy after neoadjuvant chemoradiotherapy: (A) gross residual tumor, (B) ulcer with irregular border, indicating residual tumor, $(\mathrm{C})$ ulcer with regular border, suggestive of potential complete response, and (D) telangiectasia, suggestive of complete response. 
Combination Assessment of Clinical Complete Response of Patients With Rectal Cancer Following

Chemoradiotherapy With Endoscopy and Magnetic Resonance Imaging

\section{Coloproctology Hye Mi Ko, et al}

Table 1. Definition of scores for assessment of complete response on endoscopy and MRI after chemoradiotherapy

\begin{tabular}{|c|c|c|}
\hline Score & Endoscopic findings & T2W-MRI findings \\
\hline 1 & Gross residual tumor & Gross residual isointense mass and/or involved lymph node \\
\hline 2 & Ulcer with irregular border or visible mass or polypoid tissue & Small residual isointense mass and/or involved lymph node \\
\hline 3 & Shallow ulcer with regular border and negative biopsy & $\begin{array}{l}\text { Irregular wall thickening with both hypointense and isointense signal and no involved } \\
\text { lymph nodes }\end{array}$ \\
\hline 4 & Scar, telangiectasia, erythema and negative biopsy & $\begin{array}{l}\text { Normalized rectal wall or only subtle hypointense wall thickening and no involved lymph } \\
\text { nodes }\end{array}$ \\
\hline
\end{tabular}

T2W-MRI, T2-weighted magnetic resonance imaging.

sia. These 3 cases were considered to have remnant tumors. We rated the endoscopic findings and assigned a confidence level (14) for the endoscopic assessment (Table 1).

\section{Assessment of MRI}

All patients underwent both pre- and post-CRT rectal MRI with a 1.5-T system (Signa Excite, GE Healthcare, Amersham, UK) and 3.0-T system (Achieva, Philips Medical Systems, Best, The Netherlands) using a phased array body coil. No bowel preparation was performed. To reduce colonic motility, $40 \mathrm{mg}$ of scopolamine butylbromide was injected intramuscularly before the start of the examination unless contraindicated. We also used approximately $50 \mathrm{~mL}$ of endorectal gel. The rectal MRI protocol consisted of a multiplanar conventional and high-resolution oblique T2weighted fast spin-echo sequence, axial T1-weighted sequences, and diffusion-weighted imaging. Oblique axial images were obtained orthogonal to the long axis of the tumor. Moreover, we obtained gadolinium-enhanced T1-weighted spin-echo images with fat saturation after the administration of $0.1 \mathrm{mmol} / \mathrm{kg}$ of body weight of gadolinium contrast (Omniscan, GE Healthcare).

MRI findings were examined by a gastro-intestine specialized radiologist (JEL) who had no endoscopic or pathologic information on the patients. The radiologist rated the MRI findings and assigned a confidence level (1-4) for the MRI assessment (Table 1). Fig. 2 shows the T2W-MRI findings based on the score of confidence level. Score 4 was considered to be clinical CR and/or potential CR.

\section{Statistical analysis}

Statistical analyses were carried out using IBM SPSS Statistics ver. 24.0 (IBM Co., Armonk, NY, USA). The cutoff for sensitivity, specificity, accuracy, and positive predictive value/negative predictive value of clinical CR were determined by a score was $\geq 3$ for endoscopy and a score of 4 for MRI. Pathologic CR was considered the positive outcome. Receiver operator characteristics (ROC) curves were conducted according to score assessment of endoscopy and MRI. The area under the ROC curve (AUC) was calculated for all diagnostic modalities that included combined interpretations. The positive and negative likelihood ratios were calculated directly from sensitivities and specificities for endoscopic and MRI findings [12]. These likelihood ratios were used
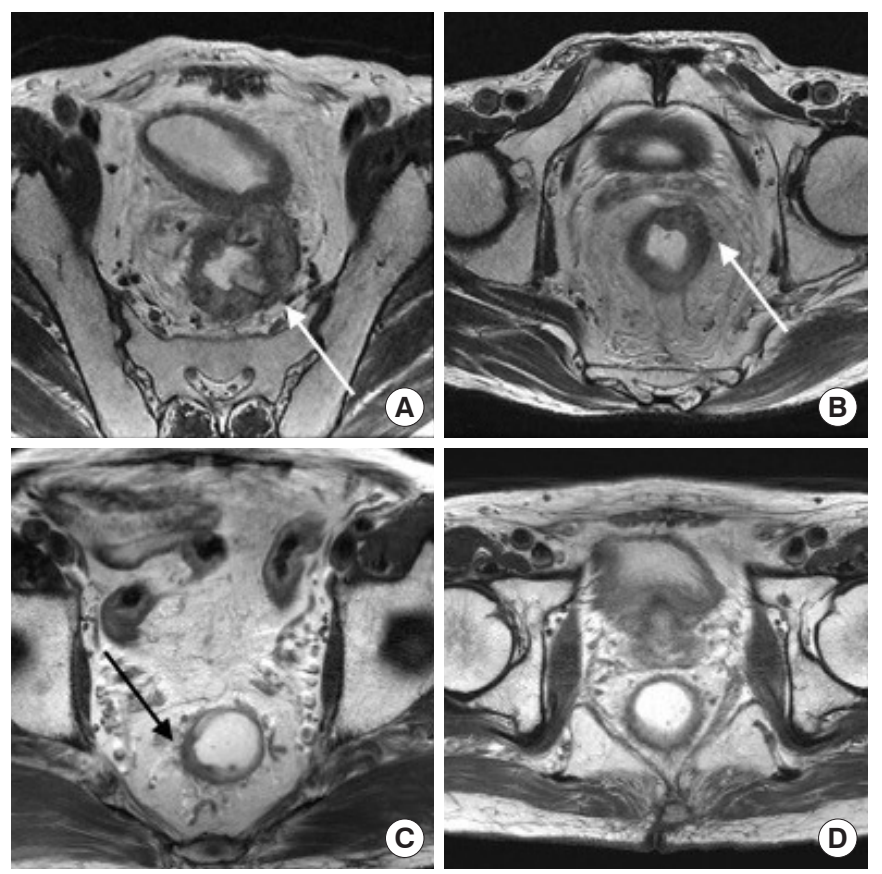

Fig. 2. Response assessment with T2-weighted magnetic resonance imaging after neoadjuvant chemoradiotherapy: (A) gross residual isointense mass (arrow), (B) small isointense mass (arrow), (C) irregular wall thickening with both hypointense and isointense signal mass, suggestive of residual tumor (arrow), and (D) normalized rectal wall, suggestive of complete response.

to calculate the posttest probabilities for CR by multiplying the pretest odds with the likelihood ratios [13]. McNemar test was used to compare the sensitivity and specificity of endoscopy, MRI, and the combination method. A P-value $<0.05$ was considered statistically significant.

\section{RESULTS}

A total of 102 patients was enrolled in this study. The clinical characteristics are presented in Table 2. There were 73 men (71.6\%) and 29 women (28.4\%), with ages from 35 to 86 years (mean, 64.6 years). Forty-seven patients $(46.1 \%)$ had lower rectal cancer, 52 patients (51.0\%) had middle rectal cancer, and 3 pa- 
Table 2. Clinical characteristics $(\mathrm{n}=102)$

\begin{tabular}{lc}
\hline Characteristic & Value \\
\hline Age (yr), mean (range) & $64.6(35-86)$ \\
$\leq 65$ & $49(48.0)$ \\
$>65$ & $53(52.0)$ \\
Sex & \\
Male & $73(71.6)$ \\
Female & $29(28.4)$
\end{tabular}

Pretreatment CEA (ng/mL)

$\begin{array}{ll}\leq 5 & 58(56.9) \\ >5 & 44(43.1)\end{array}$

Tumor location from anal verge $(\mathrm{cm})$

$\begin{array}{lc}\leq 4 & 47(46.1) \\ 4-8 & 52(51) \\ >8 & 3(2.9)\end{array}$

Histology

Well differentiated

$9(8.8)$

Moderately differentiated

89 (87.3)

Poorly differentiated

$4(3.9)$

Operative type

$\begin{array}{lc}\text { Abdominoperineal resection } & 14(13.7) \\ \text { Low anterior resection } & 81(79.4) \\ \text { Hartmann operation } & 1(1) \\ \text { Transanal excision } & 6(5.9)\end{array}$

Pathologic T stage

$\begin{array}{lc}\text { Complete remission } & 17(16.7) \\ \text { T1 } & 6(5.9) \\ \text { T2 } & 34(33.3) \\ \text { T3 } & 41(40.2) \\ \text { T4 } & 4(3.9)\end{array}$

Pathologic N stage

\begin{tabular}{cc} 
Nx & $6(5.9)$ \\
N0 & $71(69.6)$ \\
N1 & $19(18.6)$ \\
N2 & $6(5.9)$ \\
Tumor regression grade & \\
1 & $17(16.7)$ \\
2 & $24(23.5)$ \\
3 & $48(47.1)$ \\
4 & $13(12.7)$ \\
\hline
\end{tabular}

(Continued to the next)
Table 2. Continued

\begin{tabular}{lc}
\hline Characteristic & Value \\
\hline Post irradiation endoscopic finding & \\
Score 1 & $41(40.2)$ \\
Score 2 & $45(44.1)$ \\
Score 3 & $9(8.8)$ \\
Score 4 & $7(6.9)$ \\
Post irradiation T2W-MRI finding & \\
Score 1 & $54(52.9)$ \\
Score 2 & $18(17.6)$ \\
Score 3 & $14(13.7)$ \\
Score 4 & $16(15.7)$ \\
\hline
\end{tabular}

Values are presented as number (\%) unless otherwise indicated.

CEA, carcinoembryonic antigen; T2W-MRI, T2-weighted magnetic resonance imaging.

tients $(2.9 \%)$ had upper rectal cancer. Abdominoperineal resection was performed in 14 patients (13.7\%), lower anterior resection in 81 patients (79.4\%), Hartmann's operation in 1 patient (1.0\%) because of poor bowel preparation, and local excision in 6 patients (5.9\%) as they refused radical protectomy.

After pathologic examination, CR was observed in 17 patients (16.7\%). Among them, 4 patients underwent local excision. Furthermore, all patients with CR had negative lymph nodes. There were 16 patients $(15.7 \%)$ with TRG 1, 25 (24.5\%) with TRG 2, 48 (47.1\%) with TRG 3, and 13 (2.7\%) with TRG 4. Based on the endoscopic findings, there were 41 patients $(40.2 \%)$ with score 1,45 (44.1\%) with score 2, 9 (8.8\%) with score 3, and 7 (6.9\%) with score 4 . Based on the MRI finding, there were 54 patients $(52.9 \%)$ with score $1,18(17.6 \%)$ with score $2,14(13.7 \%)$ with score 3 , and $16(15.7 \%)$ with score 4.

Table 3 demonstrates the diagnostic parameters for pathologic CR of each diagnostic modality. When clinical CR was determined by a score $\geq 3$ for endoscopy, the sensitivity, specificity, and accuracy of the endoscopic evaluation were $52.9 \%, 91.8 \%$, and $85.3 \%$, respectively. When clinical CR was determined by a score of 4 for T2W-MRI, the sensitivity, specificity, and accuracy of endoscopic evaluation were $70.6 \%, 95.3 \%$, and $91.2 \%$, respectively. With the combination method, the sensitivity, specificity, and accuracy were $52.9 \%, 96.5 \%$, and $89.2 \%$, respectively. No significant differences were noted in the sensitivity and specificity of the modalities (Table 4).

The positive likelihood ratio of CR for endoscopy was 6.43, and that for T2W-MRI was 15.0. The posttest probabilities calculated from the positive likelihood ratio for the prediction of CR for endoscopy and T2W-MRI were $56.3 \%$ and $75.0 \%$, respectively. When endoscopy and T2W-MRI were combined, the posttest probability for the prediction of CR was $92.6 \%$. This indicated that the combination method could correctly predict pathologic 
Table 3. Diagnostic parameters for endoscopy, T2W-MRI, and combination

\begin{tabular}{lccc}
\hline Parameter & Endoscopy & T2W-MRI & Combination \\
\hline Sensitivity & 52.9 & 70.6 & 52.9 \\
Specificity & 91.8 & 95.3 & 96.5 \\
Accuracy & 85.3 & 91.2 & 89.2 \\
Positive predictive value & 56.3 & 75.0 & NA \\
Negative predictive value & 90.7 & 94.2 & NA \\
Positive likelihood ratio & 6.43 & 15.0 & - \\
Negative likelihood ratio & 0.51 & 0.31 & - \\
Positive posttest probability & 56.3 & 75.0 & 92.6 \\
Negative posttest probability & 9.3 & 5.8 & 8.9 \\
AUC & $0.837(0.74-0.93)$ & $0.874(0.78-0.97)$ & 0.882 (0.80-0.96) \\
\hline
\end{tabular}

Positive posttest probability is the probability of complete response (CR) when both tests have positive results, and negative posttest probability is the probability of CR when both tests have negative results. Diagnostic parameters were calculated when clinical CR was determined by score was $\geq 3$ for endoscopy and 4 for T2W-MRI. T2W-MRI, T2-weighted magnetic resonance imaging; AUC, area under the receiver operator characteristics curve.

Table 4. Comparison of diagnostic sensitivity and specificity

\begin{tabular}{ll}
\hline Sensitivity and specificity & P-value \\
\hline Sensitivity & \\
Endoscopy vs. MRI & 0.250 \\
Combination vs. endoscopy & 1.000 \\
Combination vs. MRI & 0.250 \\
Specificity & \\
Endoscopy vs. MRI & 0.375 \\
Combination vs. endoscopy & 0.125 \\
Combination vs. MRI & 1.000 \\
\hline
\end{tabular}

MRI, magnetic resonance imaging.

$\mathrm{CR}$ in the cases at a rate of $92.6 \%$, meaning only a $7.4 \%$ risk of missing residual tumor. The negative likelihood ratio was 0.51 for endoscopy and 0.31 for T2W-MRI. These values had negative posttest probabilities of 9.3\% for endoscopy and 5.8\% for T2WMRI. The combined endoscopy and T2W-MRI had a negative posttest probability of $8.9 \%$. This means that the actual CR has an $8.9 \%$ chance of being missed if endoscopy and T2W-MRI indicated a residual tumor.

Fig. 3 shows the ROC curves for endoscopy and MRI. The AUC values of endoscopy, MRI, and the combination method were $0.837,0.874$, and 0.882 , respectively.

\section{DISCUSSION}

Generally, sensitivities and specificities are used to describe the properties of diagnostic or screening tests. However, these diagnostic performances do not predict the risk of abnormalities in the test. They describe how abnormality or normality predicts a

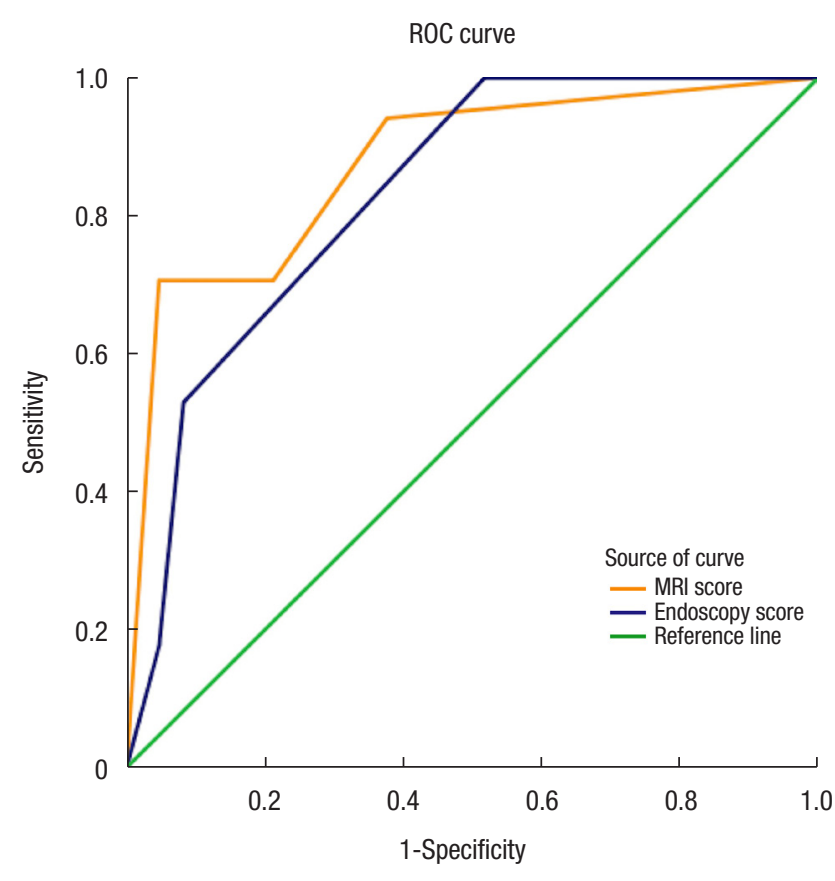

Fig. 3. Receiver operator characteristics (ROC) curves for endoscopy and T2-weighted magnetic resonance imaging. MRI, magnetic resonance imaging.

particular test result [13]. Therefore, likelihood ratios are alternative statistics for summarizing diagnostic accuracy, and they have powerful properties that make them more useful clinically than other statistics [14]. For this reason, we used the posttest probability test calculated from the likelihood ratio to evaluate the prediction of CR. In our study, T2W-MRI gave a more accurate diagnostic performance in showing pathologic CR than did endos- 
copy with biopsy, wherein the potential CR finding was considered as a prediction of the CR model. Moreover, endoscopy with biopsy demonstrated an acceptable diagnostic performance, because the sensitivity and specificity of the diagnostic modalities were not significantly different. The combination method, which involves combining endoscopy and T2W-MRI interpretations, increased the identification rate of CR and indicated a $92.6 \%$ correction rate of the cases and only $7.4 \%$ risk of missing the residual tumor.

Previous studies have reported that organ-preserving treatment, including local excision and a nonoperative strategy after neoadjuvant CRT, was feasible [7-11]. When deciding whether an organ-preserving strategy is feasible for individual patients, accurate prediction of the CR after neoadjuvant CRT is crucial. The diagnostic tools used for evaluation of CR consists of DRE, endoscopy, and imaging modalities. DRE is easy to perform and effective for identifying distal rectal cancer. However, it has been thought to underestimate the extent of rectal cancer response after neoadjuvant CRT. Given the inaccuracy of DRE following neoadjuvant CRT, it should not be used as a single tool in assessing CR or selecting patients for organ-preserving strategy following CRT [14].

Habr-Gama et al. [7, 9] used DRE and proctoscopy or colonoscopy (if possible) with biopsy to evaluate clinical CR. Although endoscopy is a very effective method for evaluating CR $[9,15]$, a major concern regarding the endoscopic assessment of response after CRT is the risk of residual tumor in the postirradiated rectum. Moreover, endoscopic biopsy had a limited clinical value for ruling out residual cancer [16]. This uncertainty of CR after CRT explains the relatively high rate of regrowth in a series of nonoperative strategies and emphasizes the need for an imaging modality that can evaluate the deeper bowel wall layer $[8,10,17]$.

The imaging modalities for assessment of response after CRT include endorectal ultrasound (ERUS), CT, and MRI. Abdominopelvic CT was used for the initial staging workup and detection of distant metastasis after CRT. However, it only had an accuracy of $33 \%$ to $77 \%$ of $\mathrm{T}$ stage for rectal cancer because of limited resolution [18]. In addition, ERUS was reported to be less accurate in assessing rectal tumor response after neoadjuvant CRT because of its dependency on the examiner, inability to perform in upper rectal cancer, and impracticality in cases of luminal narrowing [19].

Generally, post-CRT restaging MRI is performed to evaluate the mesorectal fascia in the irradiated pelvis and interval development of lymph node metastasis [20]. The accuracy of MRI restaging is lower than initial MRI staging mainly owing to the radiation effect on the rectal wall, resulting in failure to differentiate tumor infiltration from desmoplastic reaction or fibrosis. Another reason for the lower accuracy is that evaluation of mucinous adenocarcinoma on post-CRT MRI is considerably challenging because this mucinous component remains hyperintense on T2WMRI regardless of the response to CRT. However, Patel et al. [21] demonstrated treatment of 111 rectal cancer patients using neo- adjuvant CRT and concluded that T2W-MRI which is based on tumor signal intensity relative to the bowel wall layers could be a predictor for TRG and circumferential resection margin. Another observational study conducted by Lambregts et al. [22] reported that 18 out of 19 patients (94.7\%) who showed clinical CR based on T2W-MRI had consistent MRI findings during the long-term follow-up period. Hence, they concluded that MRI findings could serve as a reference for a nonoperative strategy. In our study, we used a scoring system based on morphologic change after CRT in the assessment of endoscopy and T2W-MRI. Particularly, the confidence level of T2W-MRI was based on the TRG on MRI. This might be attributed to the diagnostic performance of MRI for restaging rectal cancer after CRT, although the accuracy of MRI in response to CRT is heterogeneous in other studies [20]. The Habr-Gama study group demonstrated failure patterns for nonoperative treatment in 122 patients with clinical CR [23]. They reported that 13 patients (13.1\%) demonstrated recurrence, including 5 patients (5\%) with endorectal, 7 (7.1\%) with systemic, and $1(1 \%)$ with combined recurrence. Although endorectal recurrence could be treated with a salvage operation, the survival rate was compromised by systemic recurrence.

In the present study, the combination of endoscopy with biopsy and T2W-MRI produced a good prediction rate for pathologic CR that resulted in a $92.6 \%$ predictive value for CR. However, there is still a risk of missing remnant tumors after CRT, though all modalities indicated clinical CR. Although a good predictive value was achieved in our data, we suggest that the treatment strategy for rectal cancer after neoadjuvant CRT include radical resection with curative intent. An organ-preserving strategy such as the "wait and see" strategy or local excision should be tailored for each patient, thoroughly considering the performance status, postoperative morbidity, and life expectancy.

The main limitation of our study was that we included a relatively small number of patients with rectal cancer after CRT, and the number of patients who showed clinical or pathologic CRs was insufficient. Thus, cautious interpretation was required in the analysis. Future studies should include a large sample size to validate our novel endoscopic and T2W-MRI criteria to assess CR after CRT. Another limitation was that the time intervals between the completion of radiation, response evaluation and surgery were large, which could influence the results of the study.

In conclusion, our study demonstrated that, by combining of the interpretations from endoscopy with biopsy and T2W-MRI, a good prediction rate for $\mathrm{CR}$ in patients with rectal cancer after CRT can be established. Endoscopy with biopsy and MRI should be incorporated in restaging after CRT in rectal cancer patients when an organ-preserving treatment is being considered.

\section{CONFLICT OF INTEREST}

No potential conflict of interest relevant to this article was reported. 


\section{REFERENCES}

1. Gérard JP, Conroy T, Bonnetain F, Bouché O, Chapet O, ClosonDejardin MT, et al. Preoperative radiotherapy with or without concurrent fluorouracil and leucovorin in T3-4 rectal cancers: results of FFCD 9203. J Clin Oncol 2006;24:4620-5.

2. Sauer R, Becker H, Hohenberger W, Rödel C, Wittekind C, Fietkau $\mathrm{R}$, et al. Preoperative versus postoperative chemoradiotherapy for rectal cancer. N Engl J Med 2004;351:1731-40.

3. Onaitis MW, Noone RB, Hartwig M, Hurwitz H, Morse M, Jowell P, et al. Neoadjuvant chemoradiation for rectal cancer: analysis of clinical outcomes from a 13-year institutional experience. Ann Surg 2001;233:778-85.

4. Maas M, Nelemans PJ, Valentini V, Das P, Rödel C, Kuo LJ, et al. Long-term outcome in patients with a pathological complete response after chemoradiation for rectal cancer: a pooled analysis of individual patient data. Lancet Oncol 2010;11:835-44.

5. Stipa F, Chessin DB, Shia J, Paty PB, Weiser M, Temple LK, et al. A pathologic complete response of rectal cancer to preoperative combined-modality therapy results in improved oncological outcome compared with those who achieve no downstaging on the basis of preoperative endorectal ultrasonography. Ann Surg Oncol 2006;13:1047-53.

6. García-Aguilar J, Hernandez de Anda E, Sirivongs P, Lee SH, Madoff RD, Rothenberger DA. A pathologic complete response to preoperative chemoradiation is associated with lower local recurrence and improved survival in rectal cancer patients treated by mesorectal excision. Dis Colon Rectum 2003;46:298-304.

7. Habr-Gama A, Perez RO, Nadalin W, Sabbaga J, Ribeiro U Jr, Silva e Sousa AH Jr, et al. Operative versus nonoperative treatment for stage 0 distal rectal cancer following chemoradiation therapy: long-term results. Ann Surg 2004;240:711-8.

8. Maas M, Beets-Tan RG, Lambregts DM, Lammering G, Nelemans PJ, Engelen SM, et al. Wait-and-see policy for clinical complete responders after chemoradiation for rectal cancer. J Clin Oncol 2011;29:4633-40.

9. Habr-Gama A, Perez RO, Wynn G, Marks J, Kessler H, GamaRodrigues J. Complete clinical response after neoadjuvant chemoradiation therapy for distal rectal cancer: characterization of clinical and endoscopic findings for standardization. Dis Colon Rectum 2010;53:1692-8.

10. Smith JD, Ruby JA, Goodman KA, Saltz LB, Guillem JG, Weiser $\mathrm{MR}$, et al. Nonoperative management of rectal cancer with complete clinical response after neoadjuvant therapy. Ann Surg 2012; 256:965-72.

11. Barbaro B, Fiorucci C, Tebala C, Valentini V, Gambacorta MA, Vecchio FM, et al. Locally advanced rectal cancer: MR imaging in prediction of response after preoperative chemotherapy and radi- ation therapy. Radiology 2009;250:730-9.

12. Altman DG, Bland JM. Diagnostic tests. 1: sensitivity and specificity. BMJ 1994;308:1552.

13. Deeks JJ, Altman DG. Diagnostic tests 4: likelihood ratios. BMJ 2004;329:168-9.

14. Guillem JG, Chessin DB, Shia J, Moore HG, Mazumdar M, Bernard B, et al. Clinical examination following preoperative chemoradiation for rectal cancer is not a reliable surrogate end point. J Clin Oncol 2005;23:3475-9.

15. Kuo LJ, Chiou JF, Tai CJ, Chang CC, Kung CH, Lin SE, et al. Can we predict pathologic complete response before surgery for locally advanced rectal cancer treated with preoperative chemoradiation therapy? Int J Colorectal Dis 2012;27:613-21.

16. Perez RO, Habr-Gama A, Pereira GV, Lynn PB, Alves PA, Proscurshim I, et al. Role of biopsies in patients with residual rectal cancer following neoadjuvant chemoradiation after downsizing: can they rule out persisting cancer? Colorectal Dis 2012;14:71420.

17. Habr-Gama A, Gama-Rodrigues J, São Julião GP, Proscurshim I, Sabbagh C, Lynn PB, et al. Local recurrence after complete clinical response and watch and wait in rectal cancer after neoadjuvant chemoradiation: impact of salvage therapy on local disease control. Int J Radiat Oncol Biol Phys 2014;88:822-8.

18. Heriot AG, Grundy A, Kumar D. Preoperative staging of rectal carcinoma. Br J Surg 1999;86:17-28.

19. Pastor C, Subtil JC, Sola J, Baixauli J, Beorlegui C, Arbea L, et al. Accuracy of endoscopic ultrasound to assess tumor response after neoadjuvant treatment in rectal cancer: can we trust the findings? Dis Colon Rectum 2011;54:1141-6.

20. van der Paardt MP, Zagers MB, Beets-Tan RG, Stoker J, Bipat S. Patients who undergo preoperative chemoradiotherapy for locally advanced rectal cancer restaged by using diagnostic MR imaging: a systematic review and meta-analysis. Radiology 2013;269:10112.

21. Patel UB, Taylor F, Blomqvist L, George C, Evans H, Tekkis P, et al. Magnetic resonance imaging-detected tumor response for locally advanced rectal cancer predicts survival outcomes: MERCURY experience. J Clin Oncol 2011;29:3753-60.

22. Lambregts DM, Maas M, Bakers FC, Cappendijk VC, Lammering G, Beets GL, et al. Long-term follow-up features on rectal MRI during a wait-and-see approach after a clinical complete response in patients with rectal cancer treated with chemoradiotherapy. Dis Colon Rectum 2011;54:1521-8.

23. Habr-Gama A, Perez RO, Proscurshim I, Campos FG, Nadalin W, Kiss D, et al. Patterns of failure and survival for nonoperative treatment of stage $\mathrm{c} 0$ distal rectal cancer following neoadjuvant chemoradiation therapy. J Gastrointest Surg 2006;10:1319-28. 